\title{
Immune-related adverse kidney events by immune checkpoint inhibitors; a narrative review on current studies
}

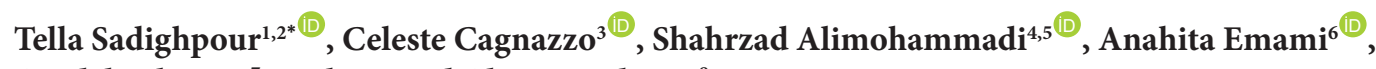 \\ Azadeh Khayyat ${ }^{7}$, Mohammad Ali EsmaeilPour ${ }^{8}$ \\ ${ }^{1}$ Florida International University, Herbert Wertheim College of Medicine, Miami, Florida, USA \\ ${ }^{2}$ American University of Antigua, College of Medicine, Antigua and Barbuda \\ ${ }^{3}$ Research and Clinical Development Unit, Pediatric Oncology and Hematology Unit, University of Turin, Turin, Italy \\ ${ }^{4}$ Doctoral School of Molecular Medicine, University of Debrecen, Debrecen, Hungary \\ ${ }^{5}$ Department of Immunology, Faculty of Medicine, University of Debrecen, Debrecen, Hungary \\ ${ }^{6}$ Independent Researcher, 12 Leadenhall Road, Toronto, Ontario, Canada \\ ${ }^{7}$ Independent Researcher 4246 Graveley St., Burnaby, BC, Canada \\ ${ }^{8}$ Adventhealth Graduate Medical Education, Center for Collaborative Research, Orlando, Florida, USA
}

\section{ART I C L E IN F O}

Article Type:

Review

\section{Article History:}

Received: 5 April 2021

Accepted: 14 May 2021

Published online: 1 June 2021

\section{Keywords:}

Immunotherapy

Glomerular filtration rate

Checkpoint inhibitors

Glomerulonephritis

Acute interstitial nephritis

Acute tubular necrosis

Acute renal failure

Immune-related adverse events

Acute tubulointerstitial nephritis

\begin{abstract}
A B S T R A C T
Chemotherapy-associated renal injury is considered one of the major concerns among nephrological and oncological practice. The use of novel anti-neoplastic therapies that target carcinomas has helped in the detection of this form of renal injury. Immune checkpoint inhibitors (ICPIs) are a group of monoclonal antibodies targeting inhibitory receptors that exist on tumor cells and $\mathrm{T}$ cells. ICPIs are able to suppress tumors that might have escaped from the immune surveillance. Meanwhile, although ICPIs have shown promising efficacy in cancer treatment, their immunerelated side effects limit their widespread use in cancer therapy schedules. One of the major side effects limiting ICPIs' usage is nephrotoxicity. Glomerular disease, acute interstitial nephritis (AIN), and acute tubular necrosis (ATN) are considered different infusion-related adverse events. Infiltration of eosinophils, T lymphocytes, and plasma cells, as well as interstitial inflammation and edema, leading to acute tubulointerstitial nephritis (ATIN). It is conceivable that the rupture of self-tolerance by ICPIs induces an autoimmune reaction against some specific self-antigens in the organs including kidneys. The exact nature of the antigen is unclear; however, it is possible that it is found in the renal tubular cells, as indicated by a greater frequency of ATIN in kidney biopsies. The current review paper discusses the relationship between ICPIs therapy and kidney disorders or more specifically, their possible role in renal damage along with renal toxicity profile in the setting of ICPIs treatment.
\end{abstract}

Implication for health policy/practice/research/medical education:

Patients treating with immune-checkpoint-inhibitors presenting with acute tubulointerstitial nephritis display a lengthy latency phase following initiation of these substances. Studies showed a milder form of acute kidney injury with milder serum creatinine improvement in contrast to acute tubulointerstitial nephritis in the background of other drugs.

Please cite this paper as: Sadighpour T, Cagnazzo C, Alimohammadi S, Emami A, Khayyat A, EsmaeilPour MA. Immunerelated adverse kidney events by immune checkpoint inhibitors; a narrative review on current studies. J Nephropharmacol. 2021;10(2):e22. DOI: 10.34172/npj.2021.22.

\section{Introduction}

Malignant cells overcome the cytotoxic immune cells' attack by downregulating their extracellular antigens, or expressing ligands that bind to the inhibitory receptors of T-cells, the immune checkpoints, and suppress T cells (1-6). Immune checkpoints are the regulatory molecules important in maintaining a balance between $\mathrm{T}$ cell activation and suppression and are required to prevent over-activation of the immune system against self-cells and resultant autoimmune disease and host organ damage (7).

Immune checkpoint inhibitors (ICPIs) are a group of monoclonal antibodies that are being utilized in the 
treatment of several malignancies, such as melanoma, lung, and kidney cancer. ICPIs prevent the binding of inhibitory receptors of T-cells and their partner ligands on tumor cells and facilitate the immune system's antitumor response (8). ICPIs are shown to have a significant clinical efficacy and currently, they are administered in the adjuvant setting for the treatment of various tumor types (9).

However, negative regulation of inhibitory checkpoints has several immune-related adverse events (irAEs), including acute kidney injury (AKI) that can progress to chronic kidneydisease (CKD), hepatitis, endocrinopathies, autoimmune colitis, and numerous cutaneous eruptions (2-5). Some studies have reported granulomatous reactions, such as sarcoidosis-like reactions, that may be misdiagnosed and the significance of which is not clear yet $(1,9-11)$.

Gupta et al estimated that the incidence of AKI in a pooled data of 3695 patients treated with ICPIs, within clinical trials was 3.3\% (8). Other investigators have also reported more or less similar rates (9-11). However, a retrospective study by Leon, reported that 272 of 920 (29.5\%) patients in a "real-world" population who received a minimum of a single dose of ICPIs developed AKI within six months of ICPIs initiation, regardless of the origin and duration of therapy (12). Leon's study showed that AKI usually presented at the $12^{\text {th }}$ to $14^{\text {th }}$ week (around three months) after initiation of therapy. However, its presentation was delayed many months to a year after ICPI therapy cessation in some cases. Additionally, other studies showed that the mortality of patients was correlated with failure of recovery from disturbed kidney function after ICPI-induced AKI (10).

Most of the biopsied kidneys of patients with ICPI usage showed acute tubulointerstitial nephritis (ATIN) as the pathology leading to AKI. ATIN may be caused by a variety of agents including IgG-4-related kidney disorders, infections, medications, and autoimmune diseases (14). Previous literature showed that ATIN provoked by ICPIs could be due to drug-specific T-cells (14) as a result of programmed cell death protein-1 (PD1) pathway inhibition followed by changing of T-cell immune tolerance (15). The current review article focuses on ICPIs' administration and the mechanisms by which they cause renal damage. Furthermore, it highlights the possible risk factors of renal toxicity in patients treated with ICPIs, as well as treatment of such cases (16).

Renal toxicity, acute renal failure, proteinuria, and electrolyte imbalance are the foremost drug-induced complications of ICPIs usage in melanoma, colorectal cancer, renal cell carcinoma, and cutaneous squamous cell carcinoma (10). Renal damage is also caused by traditional chemotherapeutic agents (11).

The time needed for symptoms to appear from the beginning of the therapy was around three months (1316).
Materials and Methods

The information provided in this review is mainly gathered from international databases including the keywords; immune checkpoint inhibitors, acute kidney injury, acute tubular necrosis, acute renal failure, glomerular filtration rate, immune-related adverse events, immunotherapy, acute tubulointerstitial nephritis, glomerulonephritis, acute kidney failure, acute interstitial nephritis, proton pump inhibitors and risk factors.

Molecular mechanisms of immune checkpoint inhibitors Immune system cells possess immune checkpoints enabling them to limit overwhelming or unrestrained activation of the immune system. Malignant tumors utilize immune checkpoints to escape from immune system attacks by binding to the inhibitory receptors of immune cells and deactivating the antitumor response. ICPIs are monoclonal antibodies that were introduced to interrupt the attachment of inhibitory receptors with ligand partners on tumor cells; therefore, increasing the function of the immune system and boosting the antineoplastic function of T-cells $(8,17)$.

The Food and Drug Administration (FDA) has approved two classes of ICPIs for use in clinical studies, cytotoxic T-cell lymphocyte-associated protein-4 (CTLA-4), and the programmed death protein-1 (PD-1)/ programmed death-ligand 1 (PD-1L) inhibitors (1). CTLA-4, PD-1, and PD-L1 are classified as inhibitory receptors of ICPIs. By inhibiting these receptors, ICPIs are capable of overcoming resistant malignant cells by boosting host immune cells to identify and get rid of tumor cells (7).

\section{An overview of renal injury by ICPIs}

The kidney is thought to be one of the most important organs affected by ICPIs. Although early reports presented a lower prevalence of renal disturbances after ICPIs administration (at around 2\%), newer studies show a $29 \%$ incidence which is considered to be relatively high (18-21). Seethapathy et al evaluated 1016 individuals with an average age of $63 \pm 13$ years ( $61 \%$ were men), with a mean serum creatinine of $0.9 \pm 0.4 \mathrm{mg} / \mathrm{dL}$ at baseline. The results revealed that a total of 169 individuals (16\%) manifested acute kidney impairment. Based on this study, the initial AKI attack developed $106 \pm 85$ days after ICPIs administration (22). A retrospective study examined renal biopsies on 16 out of 6412 cancer patients with a mean age of 64 years for 10 years after ICPI therapy. They observed that the AKI manifestations were detectable from the $6^{\text {th }}$ to $56^{\text {th }}$ weeks after the start of ICPI therapy with a median of 14 weeks. ATIN was the most common morphologic finding on renal biopsies (14 out of 16). The glomerular disorders included pauci-immune glomerulonephritis, C3 glomerulonephritis, IgA nephropathy, immune complex-mediated glomerulonephritis, and membranous glomerulopathy (9). In another study investigating the kidney biopsies of 23 metastatic melanoma patients 
with renal immune-related adverse effects (irAEs), 92\% displayed ATIN. One case of an anti-glomerular basement membrane disease (anti-GBM) disease was also diagnosed. Additionally, kidney impairment was diagnosed at a median time period of four months in individuals with renal irAEs (20).

Cortazar et al examined laboratory features and renal biopsies of 13 patients with ICPIs-induced AKI and discovered that 12 patients out of 13 , showed ATIN (19). The majority of cases presented proteinuria in a sub-nephrotic range and around half of the individuals manifested pyuria. No significant correlation was found between the peak creatinine with kidney recovery, inflammation, or fibrosis in patients affected by tubulointerstitial injury (19).

Above all, ICPI-associated acute renal failure treatment signifying severe renal injury displayed a higher mortality rate, independently (19).

Interestingly, concomitant use of proton pump inhibitors (PPIs) with ICPIs was associated with sustained acute renal impairment (22).

\section{Risk factors for ICPI-related AKI}

Risk factors for ICPI-induced kidney injury include conditions such as low-baseline glomerular filtration rate (GFR), chronic obstructive pulmonary disease, diabetes mellitus, and chronic heart disease. Moreover, some medications predispose patients to AKI upon ICPI administration, of which antibiotics, angiotensinconverting enzyme inhibitors, angiotensin-receptor blockers (ARBs), and non-steroidal anti-inflammatory drugs are the most common (21).

Seethapathy et al discovered that PPI therapy was associated with sustained renal impairment in patients treated with ICPIs (22). Cortazar et al also showed that the use of PPIs concurrently with ICPIs therapy and a lower baseline GFR were each independently associated with an increased risk of ICPI-associated AKI (19).

The mechanisms of renal toxicity due to ICPI treatment Various hypotheses have been suggested to explain the pathophysiological mechanisms of irAEs of ICPIs therapy. Cross-reactivity is one of the possible pathways. During cross-reaction, due to low selectivity, ICPI enhanced T-cells cross-react with self-antigens present on healthy tissues which may be similar to tumoral antigens (17). In addition to that, PD-1/PD-L1 appears to play a role in regulating the immune responses that help in maintaining the self-tolerance. Furthermore, ICPI therapy might disturb the immune system balance, giving rise to the production of autoantibodies that were not pathogenic in the normal state. Besides, a higher concentration of inflammatory cytokines may contribute to some morphologic lesions detected as an outcome of ICPIs therapy including podocyte foot process effacement identified in podocytopathies. Finally, direct binding of an ICPI to its specific receptor present on normal tissues will activate complement-mediated inflammation. This condition may deteriorate lesions that are already present in healthy tissue (18) since numerous antigens present on cancer cells are also expressed on normal tissues (17).

The immune-related adverse renal effects By enhancement of immune system activity, ICPIs can lead to inflammatory complications, named as "irAEs". Skin, liver, kidneys, gastrointestinal, and endocrine systems are some organs that are affected by irAEs (21). According to previous literature, the most common ICPIs-induced irAEs are skin diseases, manifesting as rash, vitiligo, and dermatitis, followed by pneumonitis, hypophysitis, colitis, and hypothyroidism.

irAEs incidence ranges from $15 \%$ to $90 \%$ (10). However, renal toxicity of ICPIs is relatively less common. The reported incidence of ICPIs-associated AKI varies from $2.2 \%$ to $29 \%$ in previous reports (16). The most common histopathological finding in patients who present with AKI due to ICPIs is ATIN with infiltration of T lymphocytes, eosinophils, and plasma cells into the interstitium, together with interstitial edema (10).

Self-reactive $\mathrm{T}$ cells are detected in a significant number of healthy individuals. The blockage of PD-1 or CTLA4 with ICPIs may jeopardize the self-tolerance state and stimulate an autoimmune reaction against some specific self-antigens in the organs including kidneys. Although the nature of antigens is not fully known, it is probable that the antigens exist on renal tubular cells considering the principal presentation of ATIN in the kidney biopsy specimens (8).

ICPI-induced AKI demonstrates relatively similar clinical and laboratory characteristics to AKI caused by other substances such as sub-nephrotic range proteinuria, bland urinalysis or sterile pyuria. However, active urine sediment can be seen if there is glomerular or vascular involvement (16,23-25).

In order to evaluate the differences between ICPIinducted AIN and non-ICPI-related AIN based on the clinical features and the renal function recovery, Draibe et al investigated 47 individuals diagnosed with AIN, of which 13 patients presented with ICPI-induced AIN, while 34 patients were diagnosed with AIN as a result of other cancer therapies. Patients diagnosed with ICPI-related AIN revealed a lower plasma creatinine level accompanied by higher urinary leucocyte counts compared with the classical AIN. The serum creatinine decrement was also lower in cases of ICPI administered patients compared to non-ICPI-related ones (26).

The morphological features of AIN were shown to be similar to those diagnosed with classical drug-induced hypersensitivity reactions (27). However, the time frame between AIN presentation and initiation of anti-cancer treatment was longer in patients with ICPI-related AIN compared to the classical AIN. AIN has been shown to 
occur up to 22 months after the administration of ICPIs and in some cases more than eight weeks after receiving the last immunotherapy dosage, which is in contrast to the conventional 7-10 days in classical acute tubular necrosis (ATN) (18). Another distinct feature of ICPI-associated AIN is that re-initiation of ICPIs does not consistently cause recurrence of renal damage (27). Based on a case report, AIN relapsed one month after corticosteroid discontinuation while other studies showed no further event after receiving ICPIs (18).

AIN is not the only pathology seen with ICPIsassociated AKI. Glomerular abnormalities have been observed with ICPIs including focal segmental glomerulosclerosis, minimal change disease, antiGBM disease, C3 glomerulonephritis, anti-neutrophil cytoplasmic antibody-negative pauci-immune crescentic glomerulonephritis, and IgA nephropathy (16). In a case study by Gallan et al three cases of kidney vasculitis and one case of pauci-immune crescentic glomerulonephritis were observed (23). A more recent case study revealed a case of renal endothelialitis during ICPIs therapy (24).

In a systematic review and meta-analysis on all published biopsy-proven cases/series, Kitchlu et al showed that pauci-immune glomerulonephritis and renal vasculitis $(27 \%)$ were the most frequent forms of glomerular disease followed by, podocytopathies (24\%), and C3 glomerulonephritis (11\%) (13).

In another study, ATN was observed in 12 cases receiving pembrolizumab treatment. Their study showed that seven of 12 renal biopsies presented ATN, and the remaining four patients presented with ATIN, while one had minimal change disease alone (28).

It is possible that the drug acts as an exogenous antigen or hapten and triggers an immune reaction when attached to the tubular antigens. It is also plausible that ATN is caused by failure of tolerance of the effector $\mathrm{T}$ cells primed during ICPIs exposure (8).

\section{Conclusion}

The efficacy of immunotherapy treatment does not relate to the structure or dosing of the medication only. Much attention is now directed to the cellular characteristics of the malignant cells as well as their interaction with immune cells and patients' tolerance.

Side effects of ICPIs, especially high incidence of AKI should be considered by oncologists and nephrologists when prescribing these agents. Early detection of ICPIassociated nephrotoxicity, including with kidney biopsy if required, will assist guaranteed and suitable management of patients with AKI after ICPI therapy.

Finally, due to the fact that patients developing ICPIinduced AIN display a lengthy latency phase following ICPIs administration, and a milder type of AKI with a mild serum creatinine improvement, it is a distinct feature and should be distinguished from the ATIN due to other drugs.

\section{Authors' contribution}

TS prepared primary draft. CC conducted the first edit. SA, AA, AK and MAE conducted further scientific edits. All authors read and signed the final manuscript.

\section{Conflicts of interest}

The authors declared no potential conflicts of interest with respect to the research, authorship, and/or publication of this article.

\section{Ethical considerations}

Ethical issues (including plagiarism, data fabrication, double publication) have been completely observed by the authors.

\section{Funding/Support}

None.

\section{References}

1. Cornejo CM, Haun P, English J, Rosenbach M. Immune checkpoint inhibitors and the development of granulomatous reactions. J Am Acad Dermatol. 2019;81:1165-75.

2. Naidoo J, Page DB, Li BT, Connell LC, Schindler K, Lacouture ME, et al. Toxicities of the anti-PD-1 and anti-PD-L1 immune checkpoint antibodies. Ann Oncol. 2015;26:2375-91.

3. O'Day SJ, Maio M, Chiarion-Sileni V, Gajewski TF, Pehamberger H, Bondarenko IN, et al. Efficacy and safety of ipilimumab monotherapy in patients with pretreated advanced melanoma: a multicenter single-arm phase II study. Ann Oncol. 2010;21:1712-7.

4. Weber JS, Kähler KC, Hauschild A. Management of immune-related adverse events and kinetics of response with ipilimumab. J Clin Oncol. 2012;30:2691-7.

5. Wolchok JD, Neyns B, Linette G, Negrier S, Lutzky J, Thomas L, et al. Ipilimumab monotherapy in patients with pretreated advanced melanoma: a randomised, doubleblind, multicentre, phase 2, dose-ranging study. Lancet Oncol. 2010;11:155-64.

6. Schreiber RD, Old LJ, Smyth MJ. Cancer immunoediting: integrating immunity's roles in cancer suppression and promotion. Science. 2011;331:1565-70. doi: 10.1126/ science.1203486.

7. Perazella MA, Shirali AC. Immune checkpoint inhibitor nephrotoxicity: what do we know and what should we do? Kidney Int. 2020;97:62-74 doi: 10.1016/ j.kint.2019.07.022.

8. Gupta S, Cortazar FB, Riella L V, Leaf DE. Immune checkpoint inhibitor nephrotoxicity: update 2020. Kidney360.2020;1:130-140. doi:10.34067/KID.0000852019.

9. Mamlouk O, Selamet U, Machado S, Abdelrahim M, Glass WF, Tchakarov A, et al. Nephrotoxicity of immune checkpoint inhibitors beyond tubulointerstitial nephritis: a single-center experience. J Immunother Cancer. 2019;7(1):2 doi: 10.1186/s40425-018-0478-8.

10. Franzin R, Netti GS, Spadaccino F, Porta C, Gesualdo L, Stallone G, et al. The use of immune checkpoint inhibitors in oncology and the occurrence of AKI: Where do we stand? Front Immunol. 2020;11:2619 doi: 10.3389/ 
fimmu.2020.574271.

11. Nicolaysen A. Nephrotoxic chemotherapy agents: old and new. Adv Chronic Kidney Dis. 2020;27:38-49. doi: 10.1053/j.ackd.2019.08.005.

12. Leon CA. Nephrotoxicity due to immune checkpoint inhibitors in rural oncology. Am Soc Clin Oncol. 2020;38: e15084. doi: 10.1200/JCO.2020.38.15_suppl.e15084.

13. Kitchlu A, Jhaveri KD, Wadhwani S, Deshpande P, Harel Z, Kishibe T, et al. A systematic review of immune checkpoint inhibitor-associated glomerular disease. Kidney Int Rep. 2020;6:66-77. doi: 10.1016/j.ekir.2020.10.002.

14. Spanou Z, Keller M, Britschgi M, Yawalkar N, Fehr T, Neuweiler J, et al. Involvement of Drug-Specific T Cells in Acute Drug-Induced Interstitial Nephritis. J Am Soc Nephrol. 2006;17:2919-27. doi:10.1681/ASN.2006050418.

15. Koda $R$, Watanabe $H$, Tsuchida $M$, Iino $N$, Suzuki $\mathrm{K}$, Hasegawa $\mathrm{G}$, et al. Immune checkpoint inhibitor (nivolumab)-associated kidney injury and the importance of recognizing concomitant medications known to cause acute tubulointerstitial nephritis: a case report. BMC Nephrol. 2018;19:48 doi:10.1186/s12882-018-0848-y.

16. Herrmann SM, Perazella MA. Immune checkpoint inhibitors and immune-related adverse renal events. Kidney Int Rep. 2020;5:1139-48. doi: 10.1016/j.ekir.2020.04.018.

17. Steinel NC, Lee EM, Viggiano D, Capasso A, Lee MW. The renal adverse effects of cancer immunotherapy. J Nephrol. 2020;33:467-81. doi: 10.1007/s40620-019-00691-2.

18. Chuva T. Nephrotoxicity in patients receiving immune checkpoint inhibitors. Port J Nephrol Hypertens. 2020;33:227-231 doi: 10.32932/pjnh.2020.01.047.

19. Cortazar FB, Marrone KA, Troxell ML, Ralto KM, Hoenig MP, Brahmer JR, et al. Clinicopathological features of acute kidney injury associated with immune checkpoint inhibitors. Kidney Int. 2016;90:638-47 doi: 10.1016/j. kint.2016.04.008

20. Hultin S, Nahar K, Menzies AM, Long G V, Fernando SL, Atkinson V, et al. Histological diagnosis of immune checkpoint inhibitor-induced acute renal injury in patients with metastatic melanoma: a retrospective case series report. BMC Nephrol. 2020;21(1):1-9. doi: 10.1186/s12882020-02044-9.

21. Meraz-Muñoz A, Amir E, Ng P, Avila-Casado C, Ragobar C, Chan $\mathrm{C}$, et al. Acute kidney injury associated with immune checkpoint inhibitor therapy: incidence, risk factors, and outcomes. J Immunother Cancer. 2020;8(1):e000467 doi: 10.1136/jitc-2019-000467.

22. Seethapathy H, Zhao S, Chute DF, Zubiri L, Oppong Y, Strohbehn I, et al. The incidence, causes, and risk factors of acute kidney injury in patients receiving immune checkpoint inhibitors. Clin J Am Soc Nephrol. 2019;14:1692-1700 doi:10.2215/CJN.00990119.

23. Gallan AJ, Alexander E, Reid P, Kutuby F, Chang A, Henriksen KJ. Renal vasculitis and pauci-immune glomerulonephritis associated with immune checkpoint inhibitors. Am J Kidney Dis. 2019;74:853-6. doi: 10.1053/j. ajkd.2019.04.016.

24. Bobart SA, Owoyemi I, Grande J, Leung N, Herrmann SM. Immune check point inhibitor-associated endothelialitis. Kidney Int Rep. 2020;5:1371-4. doi: 10.1016/j. ekir.2020.05.027.

25. Moliz C, Cavero T, Morales E, Gutiérrez E, Alonso M, Praga M. Fracaso renal agudo asociado a inhibidores check-point. Nefrología. 2020;40:206-8. doi: 10.1016/j. nefro.2019.05.004.

26. Draibe JB, García-Carro C, Martinez-Valenzuela L, Agraz I, Fulladosa X, Bolufer M, et al. Acute tubulointerstitial nephritis induced by checkpoint inhibitors versus classical acute tubulointerstitial nephritis: are they the same disease? Clin Kidney J. 2020;14:884-890. doi: 10.1093/ckj/sfaa027.

27. Sury K, Perazella MA. The nephrotoxicity of new immunotherapies. Expert Rev Clin Pharmacol. 2019;12:513-21. doi: 10.1080/17512433.2019.1613888.

28. Izzedine $\mathrm{H}$, Mathian $\mathrm{A}$, Champiat S, Picard C, Mateus C, Routier E, et al. Renal toxicities associated with pembrolizumab. Clin Kidney J. 2019;12:81-88. doi: 10.1093/ ckj/sfy 100 .

Copyright $\odot 2021$ The Author(s); Published by Published by Society of Diabetic Nephropathy Prevention. This is an open-access article distributed under the terms of the Creative Commons Attribution License (http://creativecommons.org/licenses/by/4.0), which permits unrestricted use, distribution, and reproduction in any medium, provided the original work is properly cited. 